\begin{tabular}{|c|l|}
\hline Title & Decomposition of scattering phase shifts of coupled-channels systems in the complex scaling method \\
\hline Author(s) & Odsuren, Myagmarjav; Myo, Takayuki; Kikuchi, Y uma; Teshigawara, Manabu; Kato, Kiy oshi \\
\hline Citation & $\begin{array}{l}\text { Physical Review C, 104(1),014325 } \\
\text { https://doi.org/10.1103/PhysRevC.104.014325 }\end{array}$ \\
\hline Issue Date & 2021-07-30 \\
\hline Doc URL & http://hdl.handle.net/2115/82633 \\
\hline Rights & @2021 A merican Physical Society \\
\hline Type & article \\
\hline File Information & PhysRevC.1041_014325.pdf \\
\hline
\end{tabular}

Instructions for use 


\title{
Decomposition of scattering phase shifts of coupled-channels systems in the complex scaling method
}

\author{
Myagmarjav Odsuren $\odot,{ }^{1, *}$ Takayuki Myo, ${ }^{2,3, \dagger}$ Yuma Kikuchi, ${ }^{4, \ddagger}$ Manabu Teshigawara, ${ }^{5, \S}$ and Kiyoshi Katō ${ }^{6, \|}$ \\ ${ }^{1}$ School of Engineering and Applied Sciences and Nuclear Research Center, National University of Mongolia, Ulaanbaatar 210646, Mongolia \\ ${ }^{2}$ General Education, Faculty of Engineering, Osaka Institute of Technology, Osaka 535-8585, Japan \\ ${ }^{3}$ Research Center for Nuclear Physics (RCNP), Osaka University, Ibaraki 567-0047, Japan \\ ${ }^{4}$ Tokuyama College, National Institute of Technology, Yamaguchi 745-8585, Japan \\ ${ }^{5}$ Canon Medical Systems Corporation, Otawara 324-8550, Japan \\ ${ }^{6}$ Nuclear Reaction Data Centre, Faculty of Science, Hokkaido University, Sapporo 060-0810, Japan
}

(Received 24 May 2021; accepted 16 July 2021; published 30 July 2021)

\begin{abstract}
The decomposition of scattering phase shifts in single-channel systems is extended to coupled-channels systems in the complex scaling method. The present method describes the contribution of the resonant states to the phase shift separately from the background in each channel phase shift of the coupled-channels system. We apply this method to the $\left({ }^{3} \mathrm{H}+p\right)+\left({ }^{3} \mathrm{He}+n\right)$ model for ${ }^{4} \mathrm{He}$, and show that the decomposition of phase shifts in coupled-channels systems provides useful information about the structure of scattering states.
\end{abstract}

DOI: 10.1103/PhysRevC.104.014325

\section{INTRODUCTION}

In the spectroscopic study of unstable nuclei, it is necessary to investigate the many-body resonances observed above the many-particle decay thresholds, because the unstable nuclei barely have bound states and most of the excited states are resonances [1]. Even to understand weakly bound states in unstable nuclei, such as a neutron halo nuclei, we need to investigate couplings with continuum states because of a strong coupling between them. The complex scaling method (CSM) [2] has been applied successfully to describe many-body resonant state together with weakly bound states, and we have also developed it as a powerful framework to understand the scattering phenomena of many-body systems [3-5].

In our previous paper [6], we proposed a method to decompose the scattering phase shift and cross section into resonance and background contributions in single-channel systems with the CSM. In the CSM, by solving an eigenvalue problem in a similar way as for the bound states, many-body resonant states are obtained as eigenstates with complex energies in wedge regions formed by the real energy axis and $2 \theta$ lines (rotated branch cuts) on the complex energy plane. At the same time, continuum states are obtained as being discretized along the rotated $2 \theta$ lines [2]. Using the bound, resonance, and continuum spectra obtained in the CSM, we can describe the scattering phase shift [7], and extract resonant state contributions from the background in the scattering phase shift and cross section. However, the decomposition method of

\footnotetext{
*odsuren@seas.num.edu.mn

†takayuki.myo@oit.ac.jp

${ }^{\ddagger}$ kikuchi@tokuyama.ac.jp

§mtonglacier@gmail.com

"kato-iku@gd6.so-net.ne.jp
}

the phase shift has not been established in coupled-channels systems [8], even for generalizations to coupled-channels systems that have long history $[9,10]$.

In the present paper, we show that it is possible to express the phase shift of coupled-channels systems in the form of the spectral decomposition using the CSM. This method is very promising in investigations of the coupled-channels effects in multichannel resonances, which have been known to play an important role in unbound nuclei [11].

In Sec. II, we review the CSM and the decomposition of the scattering phase shift in single channel systems, and show the extension to coupled-channels systems. In Sec. III, we apply the present method to the $\left({ }^{3} \mathrm{H}+p\right)+\left({ }^{3} \mathrm{He}+n\right)$ coupled-channels system for ${ }^{4} \mathrm{He}$, which has been discussed in previous papers [8]. The obtained results for ${ }^{3} P_{1}$ and ${ }^{3} P_{2}$ states show good agreement with the previous calculations and the experimental data, but we find a discrepancy for the ${ }^{3} P_{0}$ state. In Sec. IV, we discuss the decomposed phase shifts of the ${ }^{3} P_{0}$ state. The relation between two resonant poles of single-channel and coupled-channels systems by changing the strength factor $f$ for the coupling potential is considered. Finally, in Sec. V, the summary and conclusion will be given.

\section{DECOMPOSITION OF THE PHASE SHIFT}

\section{A. Complex scaling method}

We briefly explain the complex scaling method (CSM) proposed by Aguilar, Balslev, and Combes [2]. They introduced the transformation (dilation) $U(\theta)$ with a scaling parameter $\theta$ for the radial coordinates $r_{i}$ and momentum $\boldsymbol{k}_{i}$ of all particles $i=1, \ldots, n$ ( $n$ is the number of particles) as

$$
U(\theta) \boldsymbol{r}_{i} U^{-1}(\theta)=\boldsymbol{r}_{i} e^{i \theta}, \quad U(\theta) \boldsymbol{k}_{i} U^{-1}(\theta)=\boldsymbol{k}_{i} e^{-i \theta} .
$$


The Schrödinger equation

$$
H \Psi\left(\boldsymbol{r}_{1}, \ldots, \boldsymbol{r}_{n}\right)=E \Psi\left(\boldsymbol{r}_{1}, \ldots, \boldsymbol{r}_{n}\right),
$$

where the Hamiltonian $\mathrm{H}$ is given as

$$
H=\sum_{i=1}^{n} T_{i}-T_{c m}+\sum_{i>j}^{n} V_{i j}\left(\boldsymbol{r}_{i}-\boldsymbol{r}_{j}\right)
$$

is transformed as

$$
H(\theta) \Psi^{\theta}=E(\theta) \Psi^{\theta},
$$

where

$$
\begin{aligned}
H(\theta)= & U(\theta) H U^{-1}(\theta) \\
= & e^{-2 i \theta}\left[\sum_{i=1}^{n} T_{i}-T_{c m}\right] \\
& +\sum_{i>j}^{n} V_{i j}\left(\left(\boldsymbol{r}_{i}-\boldsymbol{r}_{j}\right) e^{i \theta}\right)
\end{aligned}
$$

and, for $f=3 n-3$,

$$
\Psi^{\theta}=U(\theta) \Psi\left(\boldsymbol{r}_{1}, \ldots, \boldsymbol{r}_{n}\right)=e^{i f \theta / 2} \Psi\left(\boldsymbol{r}_{1} e^{i \theta}, \ldots, \boldsymbol{r}_{n} e^{i \theta}\right) .
$$

Here we note that the wave function $\Psi$ is the internal one where the center-of-mass coordinate is excluded, and $f$ is the dimension of the internal coordinates. Properties of the solutions of the complex-scaled Schrödinger equation [Eq. (4)] are explained in the so-called $\mathrm{ABC}$ theorem given by Aguilar, Combes, and Balslev [2].

For simplicity, we explain the method for a single channel case of a two-particle system. Using the basis function method, where the wave function $\Psi^{\theta}$ is expanded with a finite number of $L^{2}$ basis functions, we solve an eigenvalue problem of Eq. (4). For the relative coordinate $\boldsymbol{r}$, the wave function is expressed as

$$
\Psi^{\theta}\left(\boldsymbol{r}_{1}, \boldsymbol{r}_{2}\right)=\sum_{i=1}^{N} c_{i}(\theta) \bar{u}_{i}(\boldsymbol{r}), \quad\left\langle\bar{u}_{i} \mid \bar{u}_{j}\right\rangle=\delta_{i j},
$$

where $\left\{\bar{u}_{i}(\boldsymbol{r}), i=1, \ldots, N\right\}$ is an orthonormal basis set, and the coefficients $c_{i}(\theta)(i=1, \ldots, N)$ are obtained by solving the following eigenvalue problem:

$$
\sum_{j=1}^{N}\left\langle\bar{u}_{i}|H(\theta)| \bar{u}_{j}\right\rangle c_{j}^{\alpha}(\theta)=E_{\alpha}(\theta) c_{i}^{\alpha}(\theta), \quad i=1, \ldots, N
$$

The $\mathrm{ABC}$ theorem indicates that the eigenvalues are obtained as shown in Fig. 1 and classified as

$$
\left(E_{\alpha}, \Psi^{\alpha}(\theta)\right)=\left\{\begin{array}{lll}
\left(E_{b}, \Psi^{b}\right), & b=1, \ldots, N_{b} ; & \text { bound states } \\
\left(E_{r}, \Psi^{r}\right), & r=1, \ldots, N_{r}^{\theta} ; & \text { resonant states } \\
\left(E_{c}(\theta), \Psi^{c}\right), & c=1, \ldots, N-N_{b}-N_{r}^{\theta} ; & \text { continuum states }
\end{array}\right.
$$

where $N_{b}$ and $N_{r}^{\theta}$ are the numbers of bound and resonant state solutions, respectively. The eigenvalues $E_{b}$ of the number $N_{b}$ of bound state solutions are independent of $\theta$. The resonant solutions $E_{r}$, which are obtained in the wedge region between the positive energy axis and the $2 \theta$ line as shown in Fig. 1, are also independent of $\theta$, but the number $N_{r}^{\theta}$ depends on $\theta$ because $\tan ^{-1}\left(\Gamma_{r} / 2 E_{r}^{\text {res }}\right)<2 \theta$ for $E_{r}=E_{r}^{\text {res }}-i \Gamma_{r} / 2$. The discretized energies $E_{c}(\theta)$ of continuum states are $\theta$ dependent and expressed as $E_{c}(\theta)=\epsilon_{c}^{r}-i \epsilon_{c}^{i}=\left|E_{c}\right| e^{-2 i \theta}$.

The three-kind solutions of the complex-scaled Schrödinger equation construct the extended completeness relation [12]

$$
\sum_{b=1}^{N_{b}}\left|\Psi_{b}^{\theta}\right\rangle\left\langle\widetilde{\Psi}_{b}^{\theta}\left|+\sum_{r=1}^{N_{r}}\right| \Psi_{r}^{\theta}\right\rangle\left\langle\widetilde{\Psi}_{r}^{\theta}\left|+\int_{L_{c}}\right| \Psi_{c}^{\theta}\right\rangle\left\langle\widetilde{\Psi}_{c}^{\theta}\right|=1
$$

where the tilde ${ }^{\sim}$ in bra states means the biorthogonal states with respect to the ket states, $\left\langle\widetilde{\Psi}_{\alpha}^{\theta} \mid \Psi_{\alpha^{\prime}}^{\theta}\right\rangle=\delta_{\alpha, \alpha^{\prime}}$, because of the non-Hermitian property of $H(\theta)$. The integration of the third term is taken along the $2 \theta$ line $L_{c}$ (rotated branch cut). The extended completeness relation has been proved for singlechannel and coupled-channels systems [13]. The idea of explicitly including the resonance states in the completeness relation by deforming the contour was originally proposed by Berggren [14], but in the CSM where the deformed contour is constructed by the $2 \theta$ lines, the completeness relation can be extended to multichannel and many-body systems.

In the case of eigenstates within a finite number of $L^{2}$ basis states, the integration for continuum states is approximated by

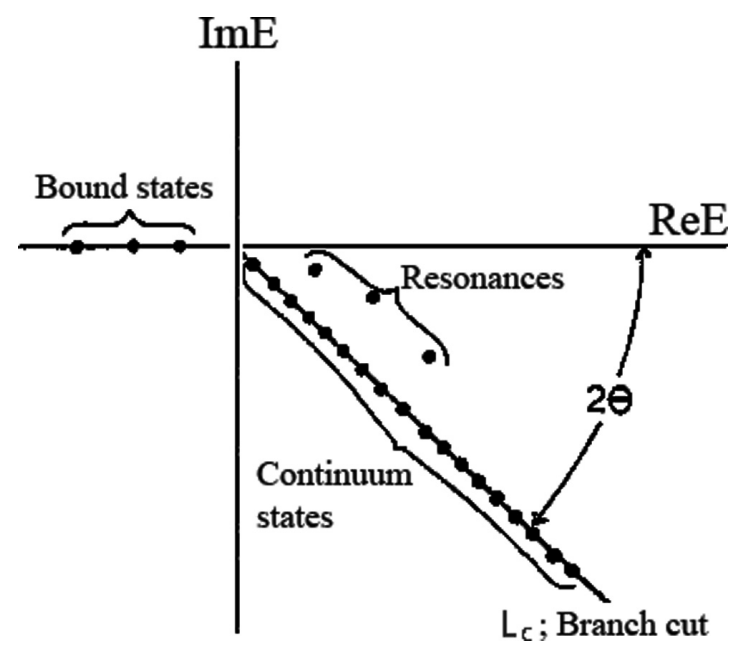

FIG. 1. The schematic eigenvalue distribution of $H(\theta)$ for a single-channel system. Continuum states on the $2 \theta$-line are discretized in the finite basis function method as solid circles. 
the summation of discretized states as

$$
\sum_{b=1}^{N_{b}}\left|\Psi_{b}^{\theta}\right\rangle\left\langle\widetilde{\Psi}_{b}^{\theta}\left|+\sum_{r=1}^{N_{r}}\right| \Psi_{r}^{\theta}\right\rangle\left\langle\widetilde{\Psi}_{r}^{\theta}\left|+\sum_{c=1}^{N-N_{b}-N_{r}}\right| \Psi_{c}^{\theta}\right\rangle\left\langle\widetilde{\Psi}_{c}^{\theta}\right| \approx 1 .
$$

It has been investigated that the reliability of the approximation of the continuum states is confirmed by using a sufficiently large basis number of $N$ in the CSM [3,4].

\section{B. Spectral decomposition of the phase shift}

The density of states at the energy $E$ is defined as

$$
\rho(E)=\operatorname{Tr}[\delta(E-H)]=-\frac{1}{\pi} \operatorname{Im} \operatorname{Tr}\left[G^{+}(E)\right] .
$$

The continuum level density (CLD) $\Delta(E)$ is given by

$$
\begin{aligned}
\Delta(E) & =\rho(E)-\rho_{0}(E) \\
& =-\frac{1}{\pi}\left\{\operatorname{Im} \operatorname{Tr}\left[G^{+}(E)\right]-\operatorname{Im} \operatorname{Tr}\left[G_{0}^{+}(E)\right]\right\},
\end{aligned}
$$

where

$$
G^{+}(E)=(E-H+i \epsilon)^{-1}, \quad G_{0}^{+}(E)=\left(E-H_{0}+i \epsilon\right)^{-1}
$$

are full and free Green's functions, respectively. The CLD is also related to the scattering matrix $S$ as shown in Ref. [15]:

$$
\Delta(E)=\frac{1}{2 \pi} \operatorname{Im} \frac{d}{d E} \ln \operatorname{det}\{S(E)\} .
$$

In the single-channel case, $S=\exp [2 i \delta(E))$, the relation between the CLD and the phase shift is expressed as

$$
\Delta(E)=\frac{1}{\pi} \frac{d \delta(E)}{d E} \text { and } \delta(E)=\pi \int_{-\infty}^{E} \Delta\left(E^{\prime}\right) d E^{\prime},
$$

Applying the extended completeness relation to calculations of Green' functions, we obtain

$$
\begin{aligned}
\Delta(E) \approx & \Delta_{\theta}^{N}(E) \\
= & -\frac{1}{\pi} \operatorname{Im}\left[\sum_{b=1}^{N_{b}} \frac{1}{E-E_{b}+i \epsilon}+\sum_{r=1}^{N_{r}^{\theta}} \frac{1}{E-E_{r}^{\mathrm{res}}+i \Gamma_{r} / 2}\right. \\
& \left.+\sum_{c=1}^{N-N_{b}-N_{r}} \frac{1}{E-\epsilon_{c}^{r}+i \epsilon_{c}^{i}}-\sum_{k=1}^{N} \frac{1}{E-\epsilon_{k}^{0 r}+i \epsilon_{k}^{0 i}}\right],
\end{aligned}
$$

where $\epsilon_{k}^{0 r}-i \epsilon_{k}^{0 i}(k=1, \ldots, N)$ are energy eigenvalues of the free Hamiltonian $H_{0}$. The approximated CLD, $\Delta_{\theta}^{N}(E)$, has a $\theta$ dependence, because basis states of a finite number $N$ are used for diagonalization of the complex scaled Hamiltonian matrix. In the calculation, we adopt a sufficiently large number of $N$ to keep numerical accuracy and to make the $\theta$ dependence negligible in the solutions. Thus, we calculate the phase shift from $\Delta_{\theta}^{N}(E)$ :

$$
\begin{aligned}
\delta_{\theta}^{N}(E)= & \int_{-\infty}^{E} d E^{\prime}\left[\sum_{b=1}^{N_{b}} \pi \delta\left(E^{\prime}-E_{b}\right)\right. \\
& +\sum_{r=1}^{N_{r}^{\theta}} \frac{\Gamma_{r} / 2}{\left(E^{\prime}-E_{r}^{\mathrm{res}}\right)^{2}+\Gamma_{r}^{2} / 4}
\end{aligned}
$$

$$
\begin{aligned}
& +\sum_{c=1}^{N-N_{b}-N_{r}} \frac{\epsilon_{c}^{i}}{\left(E^{\prime}-\epsilon_{c}^{r}\right)^{2}+\left(\epsilon_{c}^{i}\right)^{2}} \\
& \left.-\sum_{k=1}^{N} \frac{\epsilon_{k}^{0 i}}{\left(E^{\prime}-\epsilon_{k}^{0 r}\right)^{2}+\left(\epsilon_{k}^{0 i}\right)^{2}}\right] .
\end{aligned}
$$

By performing integration of every term, we obtain the spectral decomposition of the phase shift:

$$
\delta_{\theta}^{N}(E)=N_{b} \pi+\sum_{n=1}^{N_{r}^{\theta}} \delta_{r}+\sum_{c=1}^{N-N_{b}-N_{r}^{\theta}} \delta_{c}-\sum_{k=1}^{N} \delta_{k}^{0},
$$

where

$$
\cot \delta_{r}=\frac{E_{r}^{\mathrm{res}}-E}{\Gamma_{r} / 2}, \quad \cot \delta_{c}=\frac{\epsilon_{c}^{r}-E}{\epsilon_{c}^{i}}, \quad \cot \delta_{k}^{0}=\frac{\epsilon_{k}^{0 r}-E}{\epsilon_{k}^{0 i}} .
$$

\section{Coupled-channels cases}

A coupled-channels system of two-particle systems is described by using the wave function

$$
\begin{aligned}
& \Psi_{\alpha}^{\theta}(\boldsymbol{r})=\sum_{c=1}^{n_{c}} h_{c}(\hat{\boldsymbol{r}}) \psi_{c}^{\alpha}(r ; \theta), \\
& \psi_{c}^{\alpha}(r ; \theta)=\sum_{i=1}^{N_{c}} a_{c i}^{\alpha}(\theta) \bar{u}_{i}(r),
\end{aligned}
$$

where $h_{c}(\hat{\boldsymbol{r}})$ is the channel function with the channel index $c$, $n_{c}$ the number of channels, and $N_{c}$ the number of basis functions for the channel $c$. The energy spectra and radial wave functions $\psi_{c}^{\alpha}(r ; \theta)$ are obtained by solving the Schrödinger equations

$$
\begin{gathered}
H(\theta) \Psi_{\alpha}^{\theta}=E_{\alpha}(\theta) \Psi_{\alpha}^{\theta}, \\
H_{0}(\theta) \Psi_{0 \alpha^{\prime}}^{\theta}=E_{\alpha^{\prime}}^{0}(\theta) \Psi_{0 \alpha^{\prime}}^{\theta},
\end{gathered}
$$

for full and free Hamiltonians, $H$ and $H_{0}$, respectively. The indices $\alpha$ and $\alpha^{\prime}$ are to distinguish the eigenstates. Using the obtained energy spectra and wave functions, the CLD $\Delta(E)$ can be calculated as

$$
\begin{aligned}
\Delta_{\theta}^{N}(E)= & -\frac{1}{\pi} \operatorname{Im} \int d \boldsymbol{r}\langle\boldsymbol{r}|\left\{\sum_{\alpha} \frac{\left|\Psi_{\alpha}^{\theta}\right\rangle\left\langle\widetilde{\Psi}_{\alpha}^{\theta}\right|}{E-E_{\alpha}(\theta)}\right. \\
& \left.-\sum_{\alpha^{\prime}} \frac{\left|\Psi_{0 \alpha^{\prime}}^{\theta}\right\rangle\left\langle\widetilde{\Psi}_{0 \alpha^{\prime}}^{\theta}\right|}{E-E_{\alpha^{\prime}}^{0}(\theta)}\right\}|\boldsymbol{r}\rangle .
\end{aligned}
$$

Because $\left\langle\tilde{\Psi}_{\alpha}^{\theta} \mid \Psi_{\beta}^{\theta}\right\rangle=\delta_{\alpha \beta}$ and $\left\langle\widetilde{\Psi}_{0 \alpha}^{\theta} \mid \Psi_{0 \beta}^{\theta}\right\rangle=\delta_{\alpha \beta}$ for state indices $\alpha$ and $\beta$, the CLD can be expressed in a form similar to Eq. (17), while the continuum states are separated into solutions obtained on the different $2 \theta$ lines starting from the threshold energy of each channel $E_{n}^{t h}\left(n=1, \ldots, n_{c}\right)$ as shown in Fig. 2:

$$
\begin{aligned}
\Delta_{\theta}^{N}(E)= & -\frac{1}{\pi} \operatorname{Im}\left[\sum_{b=1}^{N_{b}} \frac{1}{E-E_{b}+i \epsilon}\right. \\
& +\sum_{r=1}^{N_{r}^{\theta}} \frac{1}{E-E_{r}^{\mathrm{res}}+i \Gamma_{r} / 2}
\end{aligned}
$$




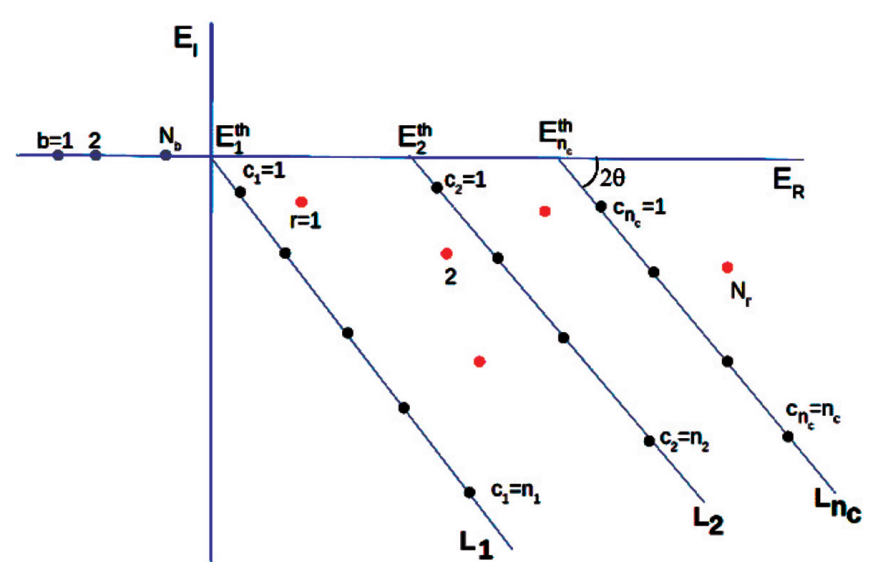

FIG. 2. The schematic eigenvalue distribution of $H(\theta)$ for a coupled-channels system in the complex energy plane. Continuum states on the $2 \theta$ lines $\left(L_{1}, L_{2}, \ldots, L_{n_{c}}\right)$ are discretized in the finite basis function method as solid circles.

$$
\begin{aligned}
& +\sum_{n=1}^{n_{c}} \sum_{c_{n}=1}^{n_{c_{n}}} \frac{1}{E-\epsilon_{c_{n}}^{r}+i \epsilon_{c_{n}}^{i}} \\
& \left.-\sum_{n=1}^{n_{c}} \sum_{k_{c_{n}}=1}^{n_{c_{n}}^{0}} \frac{1}{E-\epsilon_{k_{c_{n}}}^{0 r}+i \epsilon_{k_{c_{n}}}^{0 i}}\right],
\end{aligned}
$$

where $n_{c_{1}}+n_{c_{2}}+\cdots+n_{c_{n}}=N-N_{b}-N_{r}^{\theta}$ and $n_{c_{1}}^{0}+n_{c_{2}}^{0}+$ $\cdots+n_{c_{n}}^{0}=N$. The corresponding phase shift $\delta_{\theta}^{N}(E)$ is also obtained by integration of $\Delta_{\theta}^{N}(E)$.

Let us consider the meaning of the obtained phase shift $\delta_{\theta}^{N}(E)$ of the coupled-channels system. The $S$ matrix is unitary, described as $S_{c c^{\prime}}(E)=\exp \left(i \delta_{c c^{\prime}}\right)$, and can be diagonalized by using a real orthogonal matrix $d_{i}^{q}(E)$ :

$$
\sum_{c^{\prime}=1}^{n_{c}} S_{c c^{\prime}}(E) d_{c^{\prime}}^{q}(E)=e^{2 i \delta_{q}(E)} d_{c}^{q}(E),
$$

where $\delta_{q}(E)$ is the so-called eigenphase shift. The eigenchannel $|q\rangle$ is defined as

$$
|q\rangle=\sum_{c=1}^{n_{c}} d_{c}^{q}(E)|c\rangle, \text { inversely }|c\rangle=\sum_{q=1}^{n_{c}} d_{c}^{q}(E)|q\rangle,
$$

where $|c\rangle=\left|h_{c}\right\rangle$ is the physical channel. Thus, the CLD of the coupled-channels system, because $\ln \left[\operatorname{det}\left\{S_{c c^{\prime}}(E)\right\}\right]=i \sum_{q} \delta_{q}$ in Eq. (15), is expressed as

$$
\Delta(E)=\frac{1}{\pi} \sum_{q=1}^{n_{c}} \frac{d \delta_{q}(E)}{d E},
$$

and then the phase shift obtained by integration of $\Delta(E)$ is the sum of the eigenphase shifts:

$$
\delta_{\theta}^{N}(E)=\pi \int_{-\infty}^{E} \Delta_{\theta}^{N}\left(E^{\prime}\right) d E^{\prime}=\sum_{q=1}^{n_{c}} \delta_{q}(E) .
$$

In order to calculate each eigenphase shift $\delta_{q}(E)$, it is necessary to know $\left\{d_{c}^{q}(E)\right\}$ for the eigenchannel states.

Next, we consider how to calculate the physical-channel phase shift $\delta_{c}(E)$. In Eq. (23), from $\left\langle h_{c} \mid h_{c^{\prime}}\right\rangle=\delta_{c c^{\prime}}$ and $\left\langle\bar{u}_{i} \mid \bar{u}_{j}\right\rangle=\delta_{i j}$ we have

$$
\int d \boldsymbol{r}\left\langle\boldsymbol{r} \mid \Psi_{\alpha}^{\theta}\right\rangle\left\langle\widetilde{\Psi}_{\alpha}^{\theta} \mid \boldsymbol{r}\right\rangle=\sum_{c=1}^{n_{c}} \sum_{i=1}^{N_{c}}\left[a_{c i}^{\alpha}(\theta)\right]^{2}=\sum_{c=1}^{n_{c}}\left[A_{c}^{\alpha}(\theta)\right]^{2},
$$

where $A_{c}^{\alpha}(\theta)=\sum_{i} a_{c i}^{\alpha}(\theta)$, corresponding to the component of the channel $c$ in the total wave function, and is a complex(29 number. Therefore, Eq. (23) for the CLD is expressed as

$$
\Delta_{\theta}^{N}(E)=-\frac{1}{\pi} \operatorname{Im} \sum_{c=1}^{n_{c}}\left\{\sum_{\alpha} \frac{\left(A_{c}^{\alpha}(\theta)\right)^{2}}{E-E_{\alpha}}-\sum_{\alpha^{\prime}} \frac{\left(A_{c}^{0 \alpha^{\prime}}(\theta)\right)^{2}}{E-E_{\alpha^{\prime}}^{0}}\right\},
$$

where $\left[A_{c}^{0 \alpha}(\theta)\right]^{2}=\sum_{i}\left[a_{c i}^{0 \alpha}(\theta)\right]^{2}$ is obtained from solutions for the free Hamiltonian. Each channel term of the above expansion describes the physical-channel phase shift:

$$
\begin{aligned}
\delta_{c}(E)= & -\operatorname{Im} \int_{-\infty}^{E} d E^{\prime}\left\{\sum_{\alpha} \frac{\left[A_{c}^{\alpha}(\theta)\right]^{2}}{E^{\prime}-E_{\alpha}}\right. \\
& \left.-\sum_{\alpha^{\prime}} \frac{\left[A_{c}^{0 \alpha^{\prime}}(\theta)\right]^{2}}{E^{\prime}-E_{\alpha^{\prime}}^{0}}\right\} .
\end{aligned}
$$

When substituting eigenenergies as $E_{\alpha}=\epsilon_{\alpha}^{r}-i \epsilon_{\alpha}^{i}$ and $E_{\alpha}^{0}=$ $\epsilon_{\alpha}^{0 r}-i \epsilon_{\alpha}^{0 i}$, we can integrate on energy $E^{\prime}$ and obtain the following expression using complex variables $A_{c}^{\alpha}$ :

$$
\begin{aligned}
\delta_{c}(E)= & -\operatorname{Im}\left\{\sum_{\alpha}^{N}\left(A_{c}^{\alpha}\right)^{2}\left[\ln \left|E^{\prime}-\epsilon_{\alpha}^{r}+i \epsilon_{\alpha}^{i}\right|+i \tan ^{-1}\left(\frac{\epsilon_{\alpha}^{i}}{E^{\prime}-\epsilon_{\alpha}^{r}}\right)\right]_{-\infty}^{E}\right. \\
& \left.-\sum_{\alpha^{\prime}}^{N}\left(A_{c}^{0 \alpha^{\prime}}\right)^{2}\left[\ln \left|E^{\prime}-\epsilon_{\alpha^{\prime}}^{0 r}+i \epsilon_{\alpha^{\prime}}^{0 i}\right|+i \tan ^{-1}\left(\frac{\epsilon_{\alpha^{\prime}}^{0 i}}{E^{\prime}-\epsilon_{\alpha^{\prime}}^{0 r}}\right)\right]_{-\infty}^{E}\right\} \\
= & -\sum_{\alpha}^{N}\left\{\operatorname{Im}\left\{\left(A_{c}^{\alpha}\right)^{2}\right\} \ln \left|E-\epsilon_{\alpha}^{r}+i \epsilon_{\alpha}^{i}\right|+\operatorname{Re}\left\{\left(A_{c}^{\alpha}\right)^{2}\right\}\left[\tan ^{-1}\left(\frac{\epsilon_{\alpha}^{i}}{E-\epsilon_{\alpha}^{r}}\right)+\frac{\pi}{2}\right]\right\} \\
& +\sum_{\alpha^{\prime}}^{N}\left\{\operatorname{Im}\left\{\left(A_{c}^{0 \alpha^{\prime}}\right)^{2}\right\} \ln \left|E^{\prime}-\epsilon_{\alpha^{\prime}}^{0 r}+i \epsilon_{\alpha^{\prime}}^{0 i}\right|+\operatorname{Re}\left\{\left(A_{c}^{0 \alpha^{\prime}}\right)^{2}\right\}\left[\tan ^{-1}\left(\frac{\epsilon_{\alpha^{\prime}}^{0 i}}{E^{\prime}-\epsilon_{\alpha^{\prime}}^{0 r}}\right)+\frac{\pi}{2}\right]\right\} .
\end{aligned}
$$


When the coefficients $A_{c}^{\alpha}$ are real, the logarithmic terms will disappear. And when $A_{c}^{\alpha}=1$, we have the same expression as the single-channel case given in Eq. (19).

\section{APPLICATION TO THE $3 N+N$ MODEL}

\section{A. $3 N+N$ model for ${ }^{4} \mathrm{He}$}

We apply the present method to the $\left[{ }^{3} \mathrm{H}+p\right]+\left[{ }^{3} \mathrm{He}+n\right]$ coupled-channel model, which was proposed by Teshigawara et al. [16] to describe the ${ }^{4} \mathrm{He}$ system (isospin $T=0$ and 1). The wave function of ${ }^{4} \mathrm{He}$ with a quantum number $q={ }^{2 S+1} L_{J}$ is expressed as

$$
\left|\Psi_{q}\left({ }^{4} \mathrm{He}\right)\right\rangle=\chi_{p}^{q}(r)\left|\Phi_{p}^{q}\right\rangle+\chi_{n}^{q}(r)\left|\Phi_{n}^{q}\right\rangle
$$

where $\chi_{p}^{q}(r)$ and $\chi_{n}^{q}(r)$ are the relative radial wave functions with the relative distance $r$ for ${ }^{3} \mathrm{H}+p(p)$ and ${ }^{3} \mathrm{He}+n(n)$ channels, respectively, and $S, L$, and $J$ are the total intrinsic spin, the orbital angular momentum between $3 N$ and $N$, and the total angular momentum $(\boldsymbol{J}=\boldsymbol{L}+\boldsymbol{S})$, respectively. The channel wave functions are given by $\Phi_{p}^{q}\left({ }^{3} \mathrm{H}+p\right)$ and $\Phi_{n}^{q}$ $\left({ }^{3} \mathrm{He}+n\right)$.

The relative radial wave functions $\chi_{p}^{q}(r)$ and $\chi_{n}^{q}(r)$ are obtained by solving the Schrödinger equation

$$
H\left|\Psi_{q}\left({ }^{4} \mathrm{He}\right)\right\rangle=E\left|\Psi_{q}\left({ }^{4} \mathrm{He}\right)\right\rangle,
$$

where $H=T+V$ is the Hamiltonian given by the kinetic energy $T$ and the nuclear plus Coulomb potential $V$.

Multiplying both sides of Eq. (33) by the channel wave functions and integrating on the coordinates except for $r$, we have the coupled-channels equations

$$
\begin{array}{r}
{\left[-\frac{\hbar^{2}}{2 \mu_{p}}\left(\frac{d^{2}}{d r^{2}}+\frac{L(L+1)}{r^{2}}\right)+V_{D}^{q}(r)+\frac{e^{2}}{r} \operatorname{erf}(\sqrt{\beta} r)-E\right] \chi_{p}^{q}(r)=V_{C}^{q}(r) \chi_{n}^{q}(r),} \\
{\left[-\frac{\hbar^{2}}{2 \mu_{n}}\left(\frac{d^{2}}{d r^{2}}+\frac{L(L+1)}{r^{2}}\right)+V_{D}^{q}(r)+\Delta \epsilon-E\right] \chi_{n}^{q}(r)=V_{C}^{q}(r) \chi_{p}^{q}(r),}
\end{array}
$$

where $\beta$ is a range of the folding Coulomb potential between proton and ${ }^{3} \mathrm{H}$ cluster, and we use $\hbar^{2} / \mu_{p(n)}=$ 55.28 (55.23) $\mathrm{MeV} \mathrm{fm}^{2}$ and $\beta=0.66 \mathrm{fm}^{-2}$ in this model. The channel energy difference $\Delta \epsilon=0.763 \mathrm{MeV}$ is also used for the threshold energies of ${ }^{3} \mathrm{H}+p$ and ${ }^{3} \mathrm{He}+n$ channels in ${ }^{4} \mathrm{He}$.

The diagonal potential $\left[V_{D}^{q}(r)\right]$ and the coupling potential $\left[V_{C}^{q}(r)\right]$ are obtained from $T=1$ and $T=0$ potentials which are approximated by the single Gaussian [16]:

$$
V_{D}^{q}(r)=\frac{1}{2}\left\{V^{q, T=1} \exp \left(-\frac{r^{2}}{b_{q, T=1}^{2}}\right)+V^{q, T=0} \exp \left(-\frac{r^{2}}{b_{q, T=0}^{2}}\right)\right\}
$$

and

$$
V_{C}^{q}(r)=\frac{1}{2}\left\{V^{q, T=1} \exp \left(-\frac{r^{2}}{b_{q, T=1}^{2}}\right)-V^{q, T=0} \exp \left(-\frac{r^{2}}{b_{q, T=0}^{2}}\right)\right\} .
$$

The strengths $V^{q, T=0,1}$ and ranges $b_{q, T=0,1}$ were determined so as to reproduce the phase shift data of ${ }^{3} \mathrm{H}+n,{ }^{3} \mathrm{He}+n,{ }^{3} \mathrm{H}+p$,

\begin{tabular}{|c|c|c|c|c|c|}
\hline \multicolumn{3}{|c|}{$T=1$} & \multicolumn{3}{|c|}{$T=0$} \\
\hline$q={ }^{2 S+1} L_{J}$ & $\begin{array}{c}b_{q, T=1} \\
(\mathrm{fm})\end{array}$ & $\begin{array}{l}V^{q, T=1} \\
(\mathrm{MeV})\end{array}$ & $q={ }^{2 S+1} L_{J}$ & $\begin{array}{c}b_{q, T=0} \\
(\mathrm{fm})\end{array}$ & $\begin{array}{l}V^{q, T=0} \\
(\mathrm{MeV})\end{array}$ \\
\hline${ }^{1} \mathrm{~S}_{0}$ & 3.0 & -27.6 & ${ }^{1} \mathrm{~S}_{0}$ & 3.0 & -58.5 \\
\hline${ }^{3} S_{1}$ & 3.0 & -26.6 & ${ }^{3} \mathrm{~S}_{1}$ & 3.0 & -29.0 \\
\hline${ }^{1} P_{1}$ & 3.0 & -16.5 & ${ }^{1} P_{1}$ & & 0.0 \\
\hline${ }^{3} P_{0}$ & 1.54 & -91.54 & ${ }^{3} P_{0}$ & 2.5 & -49.5 \\
\hline${ }^{3} P_{1}$ & 3.06 & -18.83 & ${ }^{3} P_{1}$ & 3.0 & -8.0 \\
\hline${ }^{3} P_{2}$ & 3.03 & -22.93 & ${ }^{3} P_{2}$ & 3.5 & -20.0 \\
\hline
\end{tabular}
and ${ }^{3} \mathrm{He}+p$ as shown in Table I. The details of these potentials and parameters are given in Ref. [16].

Using Gaussian basis function method [17], we solve the eigenvalue problem of Eq. (34). Because the Gaussian basis

TABLE I. Parameters of the $3 N-N$ potential $V^{q, T}$. functions are not orthogonal with each other, we transform them to the orthonormal basis states which are eigenfunctions of the overlap matrix. Applying the complex scaling method (CSM), we choose the basis function parameters $\left(N, b_{0}, \gamma\right)$ [17] so as to obtain the converged solutions, where $N$ is the total number of basis functions and then $N / 2$ for each channel. The parameters $v_{i}$ of the Gaussian basis functions $\left(e^{-v_{i} r^{2}}\right)$ are given as $v_{i}=1 /\left(2 b_{i}^{2}\right)$ for $b_{i}=b_{0} \gamma^{i-1}(i=1, \ldots, N / 2)$ in the geometric progression form with $\gamma$.

\section{B. Results of ${ }^{3} \mathrm{H}+p$ and ${ }^{3} \mathrm{He}+n$ phase shifts}

In Fig. 3, we show the energy eigenvalue distribution of the ${ }^{3} P_{0},{ }^{3} P_{1}$, and ${ }^{3} P_{2}$ states obtained in the CSM with $\theta=30^{\circ}$, where $N=60, b_{0}=0.3 \mathrm{fm}$, and $\gamma=1.3$ are used. From Fig. 3(a) (left-hand side) for the ${ }^{3} P_{0}$ state, we see that the sharp resonance $E_{r}=1.13 \mathrm{MeV}$ with decay width $\Gamma=0.56 \mathrm{MeV}$ is obtained above ${ }^{3} \mathrm{He}+n$ continuum states. From Fig. 3(c) (right-hand side) for the ${ }^{3} P_{2}$ state, the broad resonance is found at $E_{r}=1.24 \mathrm{MeV}$ with decay width $\Gamma=2.32 \mathrm{MeV}$ between 

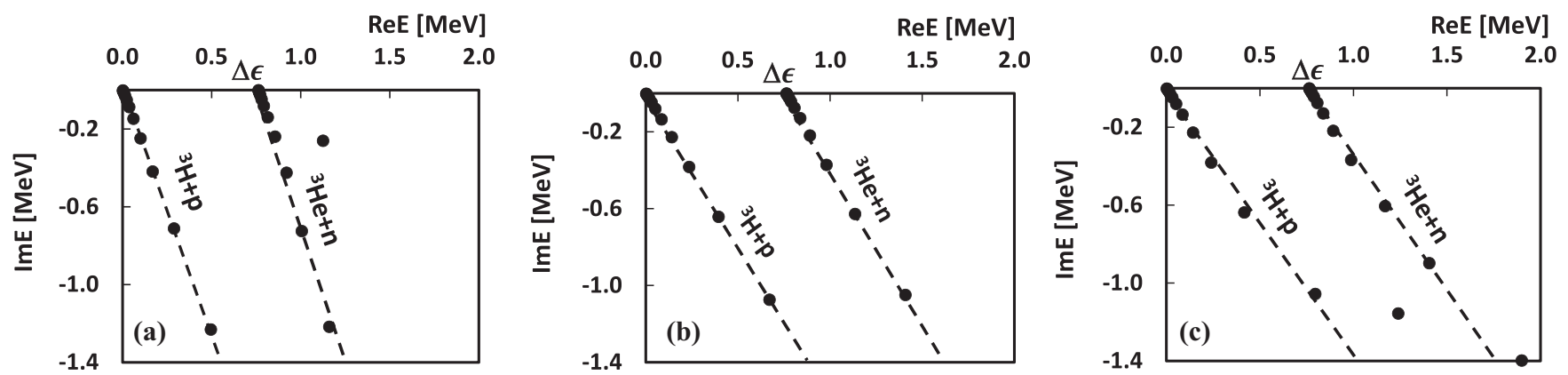

FIG. 3. Distribution of the energy eigenvalues in the complex energy plane obtained by solving the coupled-channels equation Eq. (34) with $\theta=30^{\circ}$. The filled black circles represent the calculated eigenvalues and dashed lines are $2 \theta$ lines. Left (a): ${ }^{3} P_{0}$ state. Middle (b): ${ }^{3} P_{1}$ state. Right (c): ${ }^{3} P_{2}$ state.

${ }^{3} \mathrm{H}+p$ and ${ }^{3} \mathrm{He}+n$ continuum states. The result shown in (b) of Fig. 3 (middle) indicates that the ${ }^{3} P_{1}$ state has no resonance but only continuum solutions. These results are consistent with the results of Ref. [8].

In Fig. 4, we show the results of the continuum level density (CLD) in Eq. (29) for the ${ }^{3} P_{1}$ state with different values of $\theta$. The obtained CLD has a clear stability with very small dependence on the $\theta$ values, and we cannot distinguish lines for different $\theta$ values. The total CLD [Fig. 4(a)], calculated using Eq. (29) shows the same shape as shown in Fig. 5 of Ref. [8]. In panels (b) and (c) of Fig. 4, the partial CLDs projected on ${ }^{3} \mathrm{H}+p$ and ${ }^{3} \mathrm{He}+n$ channels are shown, respectively, and also show a very small $\theta$ dependence. We can confirm that the total CLD is the sum of every channel CLD and a bending behavior of the total CLD of the ${ }^{3} P_{1}$ state at the threshold energy $\Delta \epsilon$ comes from the difference between contributions from ${ }^{3} \mathrm{H}+p$ and ${ }^{3} \mathrm{He}+n$ channel CLDs.

The phase shifts of ${ }^{3} P_{0},{ }^{3} P_{1}$, and ${ }^{3} P_{2}$ states are given in comparison with the experimental data in Fig. 5. As for the ${ }^{3} P_{2}$ states, the observed and calculated results are shown by triangles and open curves, respectively. As shown in Fig. 3(c), the ${ }^{3} P_{2}$ state has a broad resonance, and its calculated phase shifts also present broad resonance-like behaviors for both channels. Such calculated phase shift behaviors well explain the observed data for two channels of the ${ }^{3} P_{2}$ state. As can be seen from Fig. 5 , the ${ }^{3} P_{1}$ phase shifts increase smoothly in both ${ }^{3} \mathrm{H}+p$ and ${ }^{3} \mathrm{He}+n$ channels, and these results are consistent with the energy-eigenvalue distribution [see Fig. 3(b)] of no resonances. The calculated phase shifts (solid curves) for the ${ }^{3} P_{1}$ state well reproduce the experimental data (circles).

As for the ${ }^{3} P_{0}$ state, however, we see discrepancy between the calculated phase shifts (dotted lines in Fig. 5) and the experimental data (stars) in ${ }^{3} \mathrm{H}+p$ and ${ }^{3} \mathrm{He}+n$ channels. The experimental ${ }^{3} P_{0}$ phase shifts show very different behaviors for ${ }^{3} \mathrm{H}+p$ and ${ }^{3} \mathrm{He}+n$ channels. In the ${ }^{3} \mathrm{H}+p$ channel shown in Fig. 5(a), the measured phase shifts increase rapidly from $0^{\circ}$ up to $150^{\circ}$ in the energy region below the $2 \mathrm{MeV}$, and gradually go up to $210^{\circ}$ between 2 and $4 \mathrm{MeV}$. In the ${ }^{3} \mathrm{He}+n$ channel, the experimental data starting $180^{\circ}$ show a small bump at $E=1.0-1.3 \mathrm{MeV}$, and then gradually go down to $150^{\circ}$. On the other hand, the calculated ${ }^{3} P_{0}$ phase shifts show very similar resonance-like behaviors in both ${ }^{3} \mathrm{H}+p$ and ${ }^{3} \mathrm{He}+n$ channels, while they do not reach $180^{\circ}$.

\section{PHASE SHIFTS OF THE ${ }^{3} P_{0}$ STATE}

We make a detailed analysis of the calculated coupledchannels phase shifts for the ${ }^{3} P_{0}$ state to get a deep understanding the discrepancy between experiments and calculations. For this aim, we calculate the phase shifts using Eq. (31) and changing the strength of the coupling potential, and try to get useful understandings of the coupling effect of resonances on the phase shifts.

In the first step, we investigate the properties of the ${ }^{3} P_{0}$ resonant states seen in the eigenvalue distribution of Fig. 3(a). This resonant pole is expected to give a resonance behavior
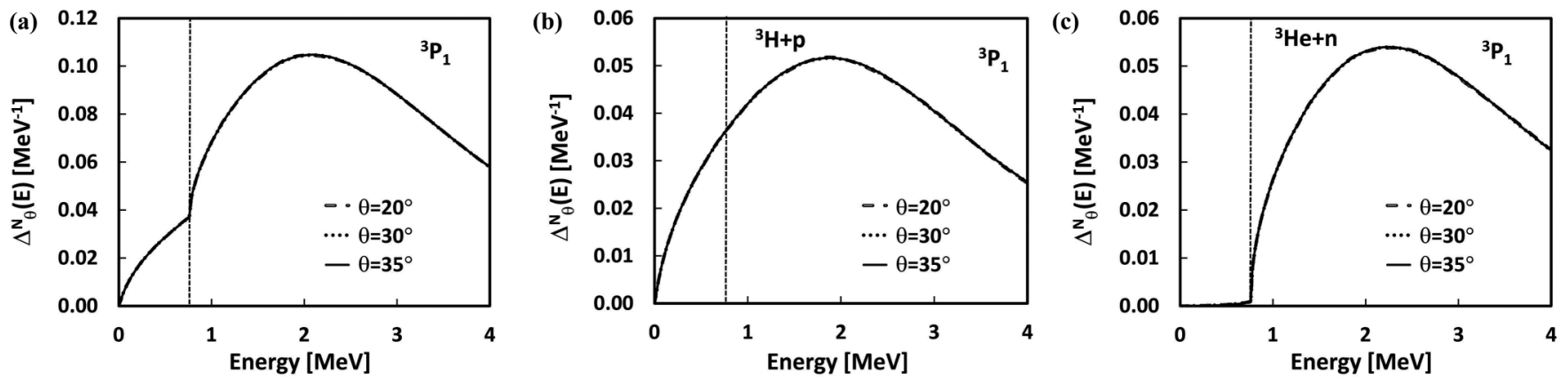

FIG. 4. The CLD of the ${ }^{3} P_{1}$ state for $\theta=20^{\circ}, 30^{\circ}$, and $35^{\circ}$ measured from the ${ }^{3} \mathrm{H}+p$ energy. (a) Left: the total CLD. (b) Middel; the ${ }^{3} \mathrm{H}+p$ channel CLD. (c) Right: the ${ }^{3} \mathrm{He}+n$ channel CLD. The vertical dashed line indicates the threshold energy of ${ }^{3} \mathrm{He}+n$ channel. 

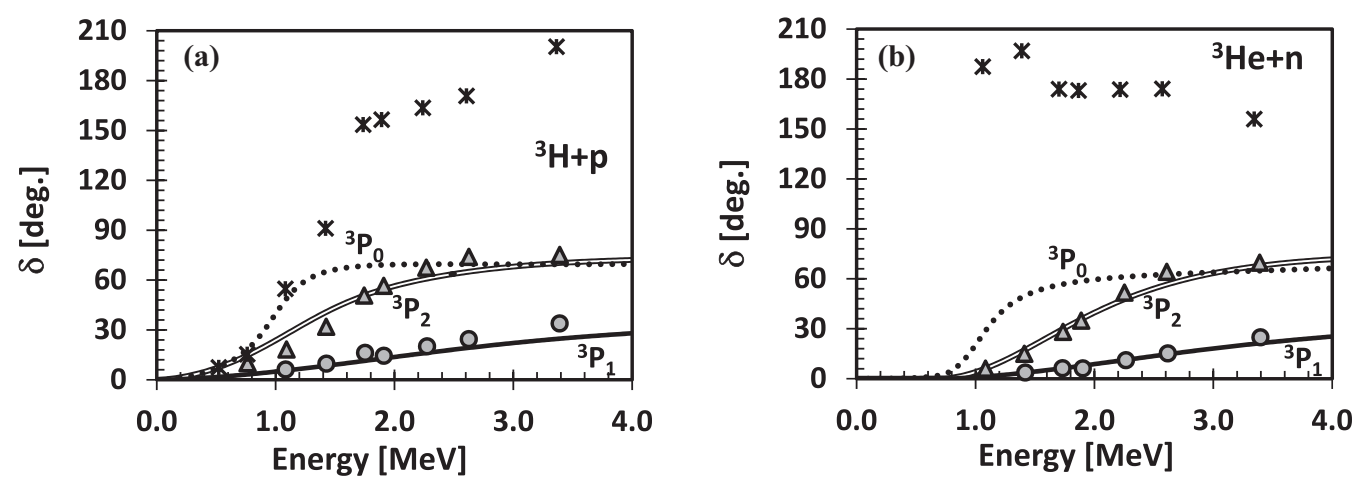

FIG. 5. The phase shifts of $P$ waves of ${ }^{3} \mathrm{H}+p$ and ${ }^{3} \mathrm{He}+n$ channels. The experimental data are taken from Ref. [18]. The observed phase shifts are displayed by stars, circles, and triangles for the ${ }^{3} P_{0},{ }^{3} P_{1}$, and ${ }^{3} P_{2}$ states, respectively. The calculated results are given by dotted $\left({ }^{3} P_{0}\right)$, solid $\left({ }^{3} P_{1}\right)$, and open $\left({ }^{3} P_{2}\right)$ curves, respectively. Left (a): the phase shifts of ${ }^{3} \mathrm{H}+p$ channel. Right (b): the phase shifts of ${ }^{3} \mathrm{He}+n$ channel.

of the observed ${ }^{3} \mathrm{H}+p$ phase shift shown in Fig. 5(a). The ${ }^{3} \mathrm{He}+n$ phase shift is seen to have a characteristic behavior different from the ${ }^{3} \mathrm{H}+p$ phase shift as shown in Fig. 5(b). To see these problems, we solve the single-channel equations for ${ }^{3} \mathrm{H}+p$ and ${ }^{3} \mathrm{He}+n$ by putting $V_{C}^{q}(r)=0$ in Eq. (34). For $q={ }^{3} P_{0}$, the potentials $V_{D}^{q}(r)$ and $V_{C}^{q}(r)$ are shown together with $V^{T=0,1}(r)$ potentials in Fig. 6 . The ${ }^{3} \mathrm{H}+p$ channel has the Coulomb potential, while the nuclear potential $V_{D}^{q}(r)$ is common in both channels. The energy eigenvalues of the complex scaled Schrödinger equations for ${ }^{3} \mathrm{H}+p$ and ${ }^{3} \mathrm{He}+n$ are presented on the same complex plane in Fig. 7(a). We find the resonances $\left(E_{1}=2.46 \mathrm{MeV}, \Gamma_{1}=4.14 \mathrm{MeV}\right)$ and $\left(E_{2}=2.79 \mathrm{MeV}, \Gamma_{2}=3.68 \mathrm{MeV}\right)$ for the ${ }^{3} \mathrm{H}+p$ and ${ }^{3} \mathrm{He}+n$ channels, respectively. They are very close to each other, because of the common nuclear potential, the small Coulomb potential, and the small threshold-energy difference.

In Fig. 7(b), we show the phase shifts calculated for singlechannel systems of ${ }^{3} \mathrm{H}+p$ (thin solid line) and ${ }^{3} \mathrm{He}+n$ (thick solid line). The two channel phase shifts have similar behav-

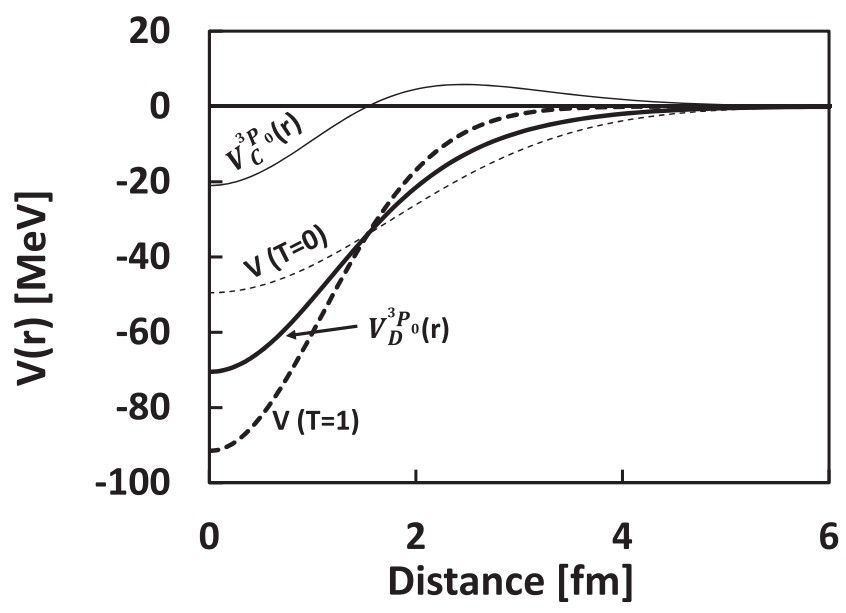

FIG. 6. The diagonal potential $\left[V_{D}^{q}(r)\right]$, the coupling potential $\left[V_{C}^{q}(r)\right]$, and the state dependence of the $3 N-N$ potential for the ${ }^{3} P_{0}$ state. The diagonal and the coupling potentials are presented by thick and thin solid curves. The potential for $T=0$ and $T=1$ states are presented by thin and thick dashed curves. ior. They start at threshold energies of ${ }^{3} \mathrm{H}+p$ and of ${ }^{3} \mathrm{He}+n$, respectively, and increase up to $\approx 90^{\circ}$ gradually. These phase shifts are decomposed into resonance and background terms by using Eq. (19) for single-channel systems [6]. The resonance and background terms are shown by broken and dotted lines, respectively. We see that the resonance terms increase up to $180^{\circ}$, but the background terms decrease gradually oppositely. These results are understood from the close position of the two poles, and the potentials are the same for both channels, except for the small Coulomb potential. The background terms show a strong repulsive behavior including the hard sphere scattering. As a result, by cancellation of the resonance and background terms, the single-channel phase shifts have a broad resonance behavior and do not reach $90^{\circ}$.

The eigenvalue distribution shown in Fig. 3(a) is obtained by the coupled-channels calculation switching on the coupling potential $V_{C}^{q}(r)$ by introducing the strength factor $f$ moving from 0 to 1 and multiplying it to $V_{C}^{q}(r)$. To see another higher resonant pole, we show an enlarged figure in Fig. 8(a). We find two resonant poles at $\left(E_{1}=1.13 \mathrm{MeV}, \Gamma_{1}=0.56 \mathrm{MeV}\right)$ and $\left(E_{2}=5.68 \mathrm{MeV}, \Gamma_{2}=11.92 \mathrm{MeV}\right)$. We also see the relation between these two resonant poles of single-channel and coupled-channels systems from Fig. 8(a), where we display traces of resonant poles calculated by changing the strength factor $f$ from zero to one for the coupling potential. The resonant poles in the single-channel calculations of the ${ }^{3} \mathrm{H}+p$ and ${ }^{3} \mathrm{He}+n$ systems go to the first and second resonant poles separately in the coupled-channels system by increasing $f$. The first resonant pole moves to lower energies and becomes sharper, and so is expected to explain the observed resonancelike phase shifts in the ${ }^{3} \mathrm{H}+p$ and ${ }^{3} \mathrm{He}+n$ channels. On the other hand, the second resonant pole is shifted up due to the coupling, and its large decay width is considered not to give a large contribution to the phase shifts observed below $4 \mathrm{MeV}$.

The properties of the coupled-channels resonances can also be seen from the channel amplitudes $A_{c}^{\alpha}$. As for the ${ }^{3} P_{0}$ solutions, we obtain

$$
\begin{aligned}
& \left(A_{3 \mathrm{H}+p}^{1 \mathrm{st}-r}\right)^{2}=0.4740+i 0.0603, \\
& \left(A_{3 \mathrm{He}+n}^{1 \mathrm{st}-r}\right)^{2}=0.5260-i 0.0603
\end{aligned}
$$



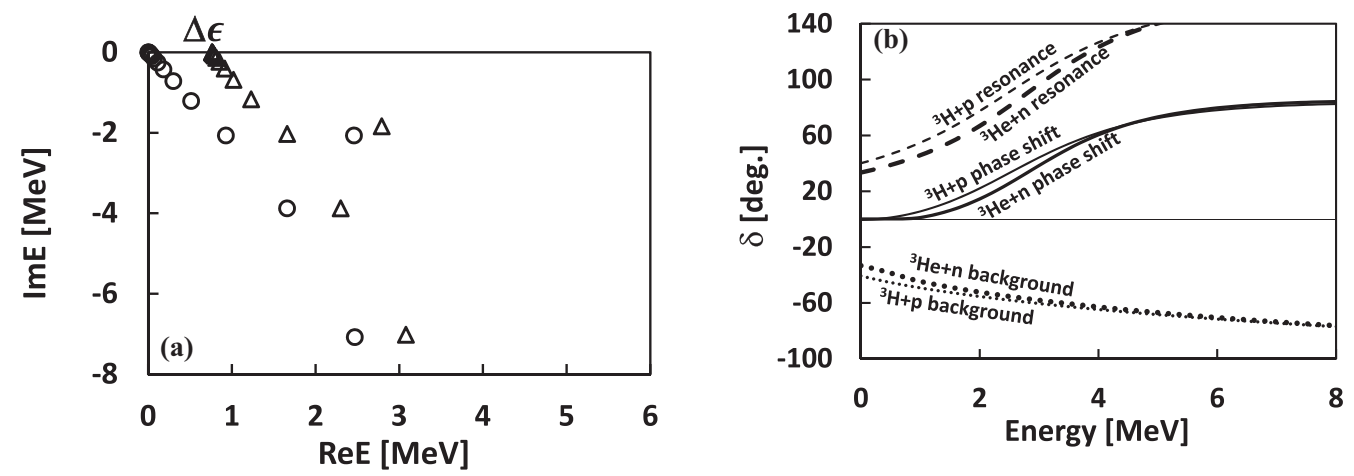

FIG. 7. The eigenvalue distribution on the complex energy plane (a) and the calculated phase shifts (b) of the ${ }^{3} P_{0}$ state calculated without the coupling potential for $\theta=30^{\circ}$. Left panel (a) displays the calculated single-channel eigenvalue distributions for ${ }^{3} \mathrm{H}+p$ (open circles) and ${ }^{3} \mathrm{He}+n$ (open triangles) channels. Right panel (b) shows the calculated singe-channel phase shifts. The contributions of the resonant poles are expressed by thin and thick dashed curves, the continuum parts are displayed by thin and thick dotted curves, and the summations of the resonant pole and continuum parts are implied by thin and thick solid curves for ${ }^{3} \mathrm{H}+p$ and ${ }^{3} \mathrm{He}+n$ channels, respectively.

for the first resonant state, and

$$
\begin{aligned}
& \left(A_{{ }^{2} \mathrm{H}+p}^{\text {nd }-r}\right)^{2}=0.5160+i 0.0056 \\
& \left(A^{2 \mathrm{He}+r}\right)^{2}=0.4840-i 0.0056
\end{aligned}
$$

for the second resonant state. The results indicate that these resonances have almost half-and-half amplitudes of the ${ }^{3} \mathrm{H}+p$ and ${ }^{3} \mathrm{He}+n$ channels due to the strong coupling. Such a strong channel coupling is explained from the fact that the resonance energies of the single channels are very close as shown in Fig. 8(a), and the coupling potential $V_{C}^{3} \mathrm{H}+p(r)$ shown in Fig. 6 is rather strong. Furthermore, the almost half-and-half amplitudes suggest that the resonant states are good isospin states of $T=0$ or 1 , and are consistent with the experimental data of ${ }^{4} \mathrm{He}$ indicating the first $0^{-}$of $T=0$ at $E_{x}=21.01 \mathrm{MeV}$ $\left(E_{r}=1.19 \mathrm{MeV}\right)$ and the second $0^{-}$of $T=1$ at $25.28 \mathrm{MeV}$ (5.46 MeV) [19].

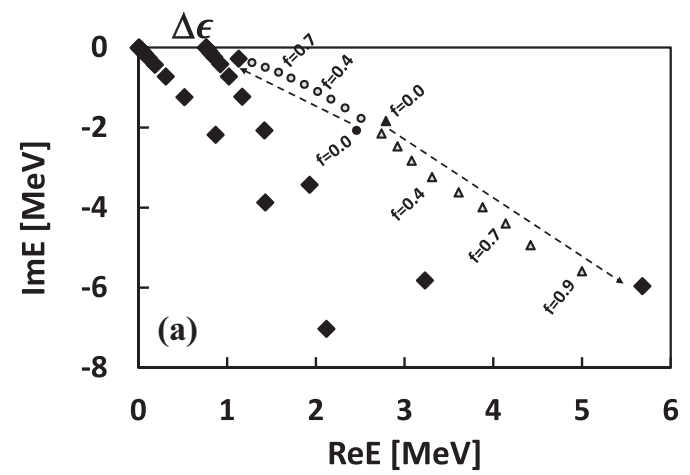

In the next step, we investigate the coupled-channels phase shifts by applying the decomposition method of Eq. (31). In the $\left({ }^{3} \mathrm{H}+p\right)+\left({ }^{3} \mathrm{He}+n\right)$ coupled-channels system, the resonance phase shift of the $\alpha$ th solution is

$$
\begin{aligned}
\delta_{c}^{\alpha}(E)= & -\operatorname{Im}\left\{\left(A_{c}^{\alpha}\right)^{2}\right\} \ln \left|E-\epsilon_{\alpha}^{r}+i \epsilon_{\alpha}^{i}\right| \\
& +\operatorname{Re}\left\{\left(A_{c}^{\alpha}\right)^{2}\right\}\left[\tan ^{-1}\left(\frac{\epsilon_{\alpha}^{i}}{E-\epsilon_{\alpha}^{r}}\right)+\frac{\pi}{2}\right],
\end{aligned}
$$

where the channel index $c$ indicates ${ }^{3} \mathrm{H}+p$ or ${ }^{3} \mathrm{He}+n$ channel. The background phase shifts of the $c$ channel are given by

$$
\delta_{\mathrm{bg}}^{\alpha}(E)=\delta_{c}(E)-\delta_{c}^{\alpha}(E) .
$$

In Fig. 8(b), the results of decomposition are shown for the two resonant states.

The first resonant state (dash-dot lines) has a sharp rising behavior similarly in both the ${ }^{3} \mathrm{H}+p$ and ${ }^{3} \mathrm{He}+n$ channels, but their resonance-like curves do not reach $180^{\circ}$. This behavior is understood from the $\left(A_{c}^{1 \text { st-r }}\right)^{2}$ amplitudes, where the

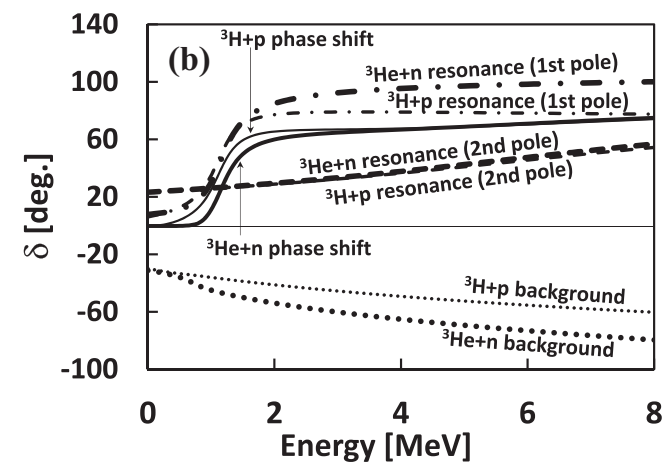

FIG. 8. The eigenvalue distribution on the complex energy plane (a) and the calculated phase shifts (b) of the ${ }^{3} P_{0}$ state with the coupling potential Eq. (36) for $\theta=30^{\circ}$. In (a) the calculated coupled-channels eigenvalue distribution is expressed by solid diamonds. The open circles and open triangles present the first and second resonant poles obtained by changing the strength factor $f$ for the coupling potential, respectively. The arrows show the pole trajectory of the two resonant poles at different strength factor $f$ for the coupling potential. In (b) the decomposition of the calculated coupled-channels phase shifts is presented. The contributions of the resonant poles are expressed by thin and thick dotted dashed and dashed curves, the continuum parts are displayed by thin and thick dotted curves, and the summations of the resonant poles and continuum parts are implied by thin and thick solid curves for ${ }^{3} \mathrm{H}+p$ and ${ }^{3} \mathrm{He}+n$ channels, respectively. 
real parts are almost half-and-half amplitudes in the ${ }^{3} \mathrm{H}+p$ and ${ }^{3} \mathrm{He}+n$ channels and the logartihmic terms in Eq. (37) are negligible because of very small imaginary values of $\left(A_{c}^{1 \mathrm{st}-r}\right)^{2}$. The second broad resonance (broken lines) has slowly increasing behavior in the ${ }^{3} \mathrm{H}+p$ and ${ }^{3} \mathrm{He}+n$ channels commonly. We cannot distinguish two thick and thin broken lines describing the contributions of different channels. Thus, the obtained second resonance is considered to give no effective contributions to the low energy phase shift behavior as well, as described above. From Fig. 8(b), we also see the background terms (dotted lines) of each channel, which are defined by subtraction of the first and second resonance contributions from the phase shift $\delta_{c}$. The repulsive background terms show gentle energy dependence and seem to cancel the second resonance contributions. As a result, the total phase shifts of the ${ }^{3} P_{0}$ state (solid lines) shows a mild resonance behavior that do not reach $90^{\circ}$ in both ${ }^{3} \mathrm{H}+p$ and ${ }^{3} \mathrm{He}+n$ channels, which is not consistent with the experimental data.

\section{SUMMARY AND CONCLUSION}

With the development of research into unstable nuclei, it has become necessary to study the unbound states of nuclear many-body systems at the same time as the bound states. For this aim, the complex scaling method (CSM) has been successfully applied so far. We have also emphasized that CSM is very effective not only for the study of many-body resonant states but also for the study of many-body scattering states $[3,4]$. For example, the contribution of the three-body resonant state in the three-body breakup reactions [20,21] and the spectral decomposition of the two-body scattering phase shifts [6] can be mentioned.

In this paper, we tried the extension of the spectral decomposition of two-body single-channel scattering phase shifts to coupled-channels cases, and proposed a decomposition expression by using energy eigenvalues and channel amplitudes of solutions in the eigenvalue problem for the complex-scaled Hamiltonian. This method was applied to the $\left({ }^{3} \mathrm{H}+p\right)+\left({ }^{3} \mathrm{He}+n\right)$ coupled-channels model for ${ }^{4} \mathrm{He}$, which was successfully developed in Ref. [16]. We confirmed that the present method and the $\left({ }^{3} \mathrm{H}+p\right)+\left({ }^{3} \mathrm{He}+n\right)$ coupledchannels model work well by comparing with the previous calculations [7,8] and the experimental data [18]. However, we find that the experimental ${ }^{3} P_{0}$ phase shifts are inconsistent with the present calculations.

To understand the reason why the calculation of the ${ }^{3} P_{0}$ phase shifts shows results different from the experimental data, we analyzed the resonance solutions and the ${ }^{3} P_{0}$ phase shifts by applying the present decomposition method in the CSM. In the present ${ }^{3} \mathrm{H}+p$ and ${ }^{3} \mathrm{He}+n$ coupled-channels model, we calculate two ${ }^{3} P_{0}$ state resonances which correspond to observed two $0^{-}$resonant states in ${ }^{4} \mathrm{He}$ [19]. From the single-channel and coupled-channels calculations, we find that the two resonances in the single channel are strongly mixed in the coupled channels: one as a sharp resonance at low energy and the other as a wide resonance at high energy. The low-energy phase shifts above the threshold energies are dominated by the first resonant state that has fifty-fifty amplitudes of ${ }^{3} \mathrm{H}+p$ and ${ }^{3} \mathrm{He}+n$ channels. These results indicate that the ${ }^{3} \mathrm{H}+p$ and ${ }^{3} \mathrm{He}+n$ channel phase shifts have very similar behavior in the low energy region.

Thus this method of the decomposition of the coupledchannels phase shifts is very useful in understanding the behavior of phase shifts in association with resonant states and their structures. In these calculations, we employed the ${ }^{3} \mathrm{H}+p$ and ${ }^{3} \mathrm{He}+n$ coupled-channels model using the $3 N-N$ potentials previously developed [16]. It is an interesting problem to search the appropriate $3 N-N$ potentials to explain the observed ${ }^{3} P_{0}$ phase shifts [18].

\section{ACKNOWLEDGMENTS}

This work was supported by JSPS KAKENHI Grants No. 17K05430, No. 20K03962, and No. 18K03660, and by the National University of Mongolia's Support for High Impact Research program P2019-3709.
[1] T. Nakamura, H. Sakurai, and H. Watanabe, Prog. Part. Nucl. Phys. 97, 53 (2017).

[2] J. Aguilar and J. M. Combes, Commun. Math. Phys. 22, 269 (1971); E. Balslev and J. M. Combes, ibid. 22, 280 (1971).

[3] S. Aoyama, T. Myo, K. Katō, and K. Ikeda, Prog. Theor. Phys. 116, 1 (2006).

[4] T. Myo, Y. Kikuchi, H. Masui and K. Katō, Prog. Part. Nucl. Phys. 79, 1 (2014).

[5] T. Myo and K. Katō, Prog. Theor. Exp. Phys. 2020, 12A101 (2020).

[6] M. Odsuren, K. Katō, M. Aikawa, and T. Myo, Phys. Rev. C 89, 034322 (2014).

[7] R. Suzuki, T. Myo, and K. Katō, Prog. Theor. Phys. 113, 1273 (2005).

[8] R. Suzuki, A. T. Kruppa, B. G. Giraud, and K. Katō, Prog. Theor. Phys. 119, 1949 (2008).

[9] R. G. Newton, Scattering Theory of Waves and Particles (Springer, New York, 1982).
[10] J. R. Taylor, Scattering Theory: The Quantum Theory of Nonrelativistic Collisions (Dover, Mineola, NY, 1983).

[11] K. Hagino, H. Sagawa, S. Kanaya, and A. Odahara, Prog. Theor. Exp. Phys. 2020, 023D01 (2020).

[12] T. Myo, A. Ohnishi, and K. Katō, Prog. Theor. Phys. 99, 801 (1998).

[13] B. G. Giraud and K. Katō, Ann. Phys. (NY) 308, 115 (2003); B. G. Giraud, K. Katō, and A. Ohnishi, J. Phys. A 37, 11575 (2004).

[14] T. Berggren, Nucl. Phys. A 109, 265 (1968).

[15] R. D. Levine, Quantum Mechanics of Molecular Rate Processes (Dover, Mineola, NY, 1969).

[16] M. Teshigawara, K. Katō, and G. F. Filippov, Prog. Theor. Phys. 92, 79 (1994)

[17] E. Hiyama, Y. Kino, and M. Kamimura, Prog. Part. Nucl. Phys. 51, 223 (2003). 
[18] I. Y. Barit and V. A. Sergeev, Sov. J. Nucl. Phys. 13, 708 (1971).

[19] Nuclear Data Evaluation Project, Triangular Universities Nuclear Laboratory (TUNL), https://nucldata.tunl.duke.edu/.
[20] T. Myo, K. Katō, S. Aoyama, and K. Ikeda, Phys. Rev. C 63, 054313 (2001).

[21] T. Myo, S. Aoyama, K. Katō, and K. Ikeda, Phys. Lett. B 576, 281 (2003). 